\title{
Creation of Closed Ecological Life Support Systems: Results, Critical Problems and Potentials
}

\author{
Iosef I. Gitelson ${ }^{\mathrm{a}, \mathrm{b}}$ * and Genry M. Lisovsky ${ }^{\mathrm{a}}$ \\ ${ }^{a}$ Institute of Biophysics of Siberian Branch of Russian Academy of Sciences, \\ Akademgorodok, Krasnoyarsk, 660036 Russia \\ ${ }^{b}$ Siberian Federal University, Svobodny av. 79, Krasnoyarsk, 660041 Russia ${ }^{1}$
}

Received 1.09.2007, received in revised form 1.12.2007, accepted 15.01.2008

\begin{abstract}
"Humanity as a whole is growing into a powerful geological force. And now humanity, with its intellect and labor, faces the problem of restructuring the biosphere for the benefit of freethinking humanity as a single whole. This new state of the biosphere, which we approach unaware of it, is the noosphere."
\end{abstract}

V. Vernadsky

As experiments have shown, a closed ecological life support system based on a biological material exchange is fully realizable and contains possibilities for further improvement. This kind of closed human ecosystem can become a model not only of an earthly noosphere, but also of daughter noospheres that can be used to help humanity render space inhabitable without threatening other bodies of the solar system through the intrusion of earthly substances and biological autocatalytic processes. As such, it will allow humans to exist in space or on other solar bodies, while requiring only the input of energy and not allowing the release of metabolites into the surroundings. Partially closed technologies for regenerating the atmosphere, water, and vegetable nutrients can radically enhance the quality of life in extreme regions - in the Arctic, Antarctica, in deserts, and in high mountain settlements. Another aspect of closed life support technologies is that they enable the minimizing of the environmental pollution that results from human waste products and those of their domestic animals. That these technologies can find applications on Earth before human settlements appear on Mars or the Moon. The transition to essentially closed nonpolluting, non-deadlock life support technologies will be a noticeable step on the path toward the sustainable development of the noosphere.

Keywords: Biosphere, Noosphere, Life support, Closed ecological system, Chlorella, algal continues cultivation.

\section{From Bios-1 to Bios-3. Results}

For nearly half a century, Kirensky - Terskov

- Gitelson's school (the youngest founder being a co-author of this article) has been pursuing a direction of research that can be defined as parametric control of biosynthesis in continuous cultures of cell populations and construction of closed ecological systems based on them.

Corresponding author E-mail address: gitelson@ibp.ru

(C) Siberian Federal University. All rights reserved 
The work started in the Institute of Physics of the Siberian Branch of the Academy of Sciences is now continued in the Institute of Biophysics SB RAS, formerly a division of the Institute of Physics, and in the Krasnoyarsk State University (now Siberian Federal University), which has been traditionally related to the Institute scientifically and educationally. The establishment of the Siberian Federal University has offered new potential opportunities for the development of this line of research and for wide practical application of the mature results. It is time now to sum up what has been accomplished and to outline the ways in which to develop ${ }^{1}$. In this paper we limit ourselves to the direction that has led to the creation of experimental closed ecological human life support systems.

The ideal foundations of our work were V.I. Vernadsky's (1924) concept of the biosphere and K.E. Tsiolkovsky's $(1926,1964)$ prediction about the feasibility of supporting human life outside Earth by biological means.

Experimental closed ecosystems are based on methodology of continuous culture. The cultured objects are unicellular microorganisms, mostly photo- and chemoautotrophic ones, and higher agricultural plants.

The installation for maintaining continuous growth and reproduction of cells, whether they are microorganism populations or clones of cells harvested from the tissues of multicellular animals or plants, consists of the biological part - growing cells - and the technical part - a system for gathering data from sensors monitoring the state of the cells and parameters of the medium; a control unit, which makes decisions based on the received data; and an execution unit, which maintains the parameters of the medium at specified levels and compensates for perturbations introduced by growing cells. The speed of growth become maximal. For instance, the reproduction rate we obtained for Photobacteria sp. was 8-12 $\min$.

If there is no preset limit to the number of replications in the genome of the cultured species, which is true for the most studied species of microorganisms (the situation being more complicated for clones of cells from tissues of multicellular organisms), this system is not limited in time and the rates of growth and reproduction of the cell population in it can be maintained over indefinitely long periods of time; theoretically this system is eternal and practically its existence is limited by the time of functioning of its technical unit.

We realized this system for the Chlorella population, optimized the physical parameters of the culture and the composition of the nutrient medium, and obtained continuous culture that was only limited by energy - by the amount of the incident photosynthetically active radiation. It produced so much oxygen that 17 liters of the culture spread in a thin layer in the reactor with the light-receiving surface area $8 \mathrm{~m}^{2}$ was enough for gas exchange of a human subject whose body mass amounted to $70 \mathrm{~kg}$. That was the basis for constructing Bios-1, a system in which the sealed cabin with the human subject was connected to the Chlorella reactor Bios-1 (Fig. 1) and the subject stayed there for days, due to gas exchange with the algae, which took up the carbon dioxide he released.

The next step was the construction of Bios2 , a system in which the water cycle was closed in a loop with the algal culture. See schematic diagram on Fig. 2.

Microalgae, however, cannot be completely responsible for the most complex biochemical

\footnotetext{
Even an overview of the main branches and fruits of this prolific line of research cannot be made within one article. We hope that the Editorial Board of the Journal will invite the leaders of the mature and the promising branches of closed ecological systems studies to resume the tradition of writing analytical reviews of their research and publish them in the Journal.
} 


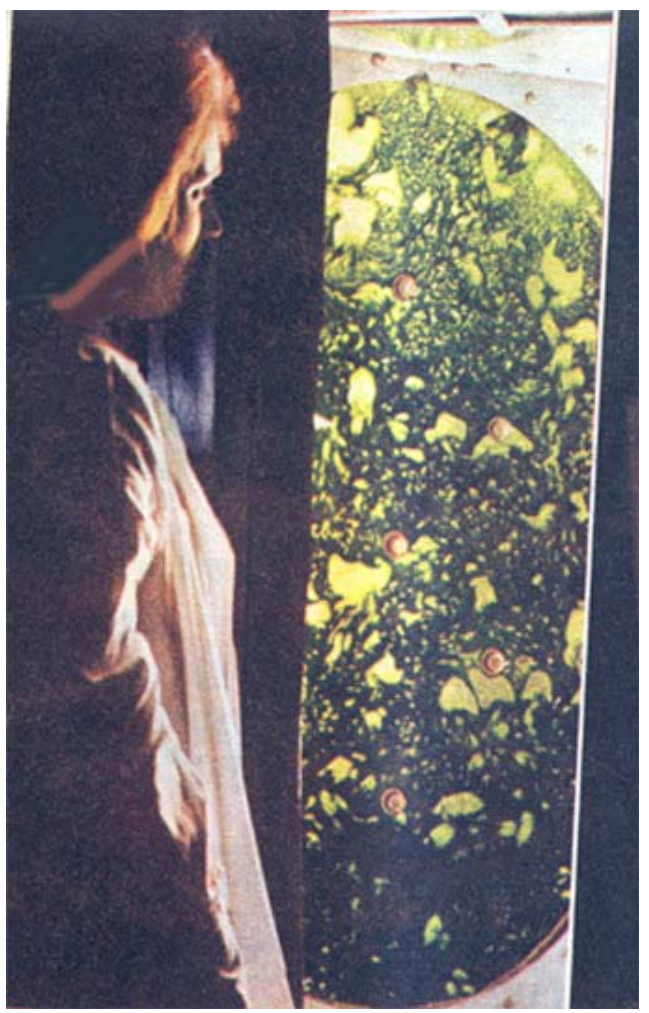

Fig. 1. Chlorella's cultivator

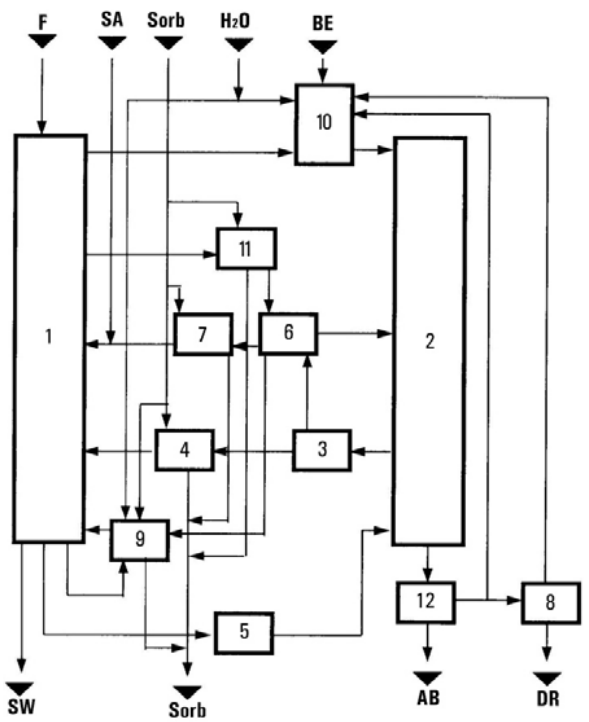

Fig. 2. Schematic showing mass exchanges in the system "human - microalgae". 1 - sealed cabin; 2 - algal cultivator; 3 - heat exchanger; 4 - charcoal filter; 5 - compressor; 6 - metering pump for condensate and waste water; 7 - filter for drinking water; 8 - distillation unit; 9 - filter for hygiene water; 10 - nutrient solution tank; 11 - filter for waste water; 12 - centrifuge. F — food; SA - salt additives to drinking water; Sorb - sorbents; $\mathrm{H}_{2} \mathrm{O}$ - water added to the system to compensate for process losses and water expenditure in algal photosynthesis; $\mathrm{BE}$ - biogenic elements; $\mathrm{SW}$ - human solid wastes; $\mathrm{AB}$ - algal biomass; DR — dry residue. 
function of the ecological human life support system - food production. Their contribution can amount to just $5-10 \%$, as their biomass mainly contains proteins and nucleic acids, while normal human diet must predominantly contain carbohydrates.

The way to provide humans with food in the closed ecosystem that was the most physiologically sound and safe for humans, at least in the first phase, was cultivation of traditional agricultural crops. One of the authors of this paper (Lisovsky, 1979) showed that higher plants grown in continuous culture - as a "conveyor" - yield, under optimal conditions, the same amount of biomass and, hence, the same amount of oxygen as the microalgal culture. It had been earlier assumed that productivity of the algae was many times higher than that of higher plants. However, we managed to provide one human with the vegetable part of his diet by growing plants on $30 \mathrm{~m}^{2}$ of the illuminated area. That was the basis for constructing Bios-3, the system designed by B.G. Kovrov et al. (1985).

Bios-3 (Fig.3) is constructed of welded stainless steel plates to provide a hermetic seal. The structure (Fig. 2), which is $14 \times 9 \times 2.5 \mathrm{~m}$ (with a volume of $315 \mathrm{~m}^{3}$ ), is divided equally into four compartments (of nearly 7 x 4.5 × $2.5 \mathrm{~m}$ ) (Fig. 4). Each compartment has three doors that are sealed tightly with rubber gaskets. One door in each compartment leads to the outside, and occupants could escape within 20 seconds if necessary, but the need has never arisen. Each compartment can be scaled independently in combination with any other compartment. The crew area, which occupies one compartment, is subdivided into three separate sleeping rooms, a kitchen, a lavatory, a control room, and a work
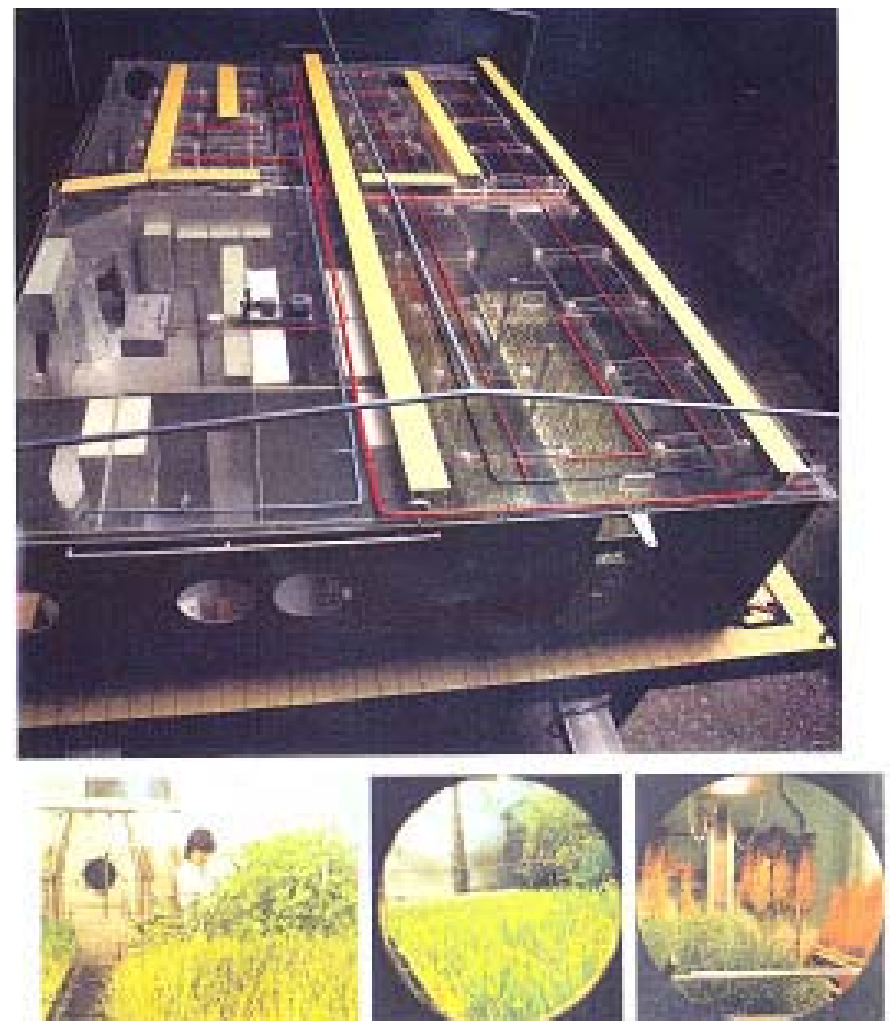

Fig. 3. Bios-3 model 


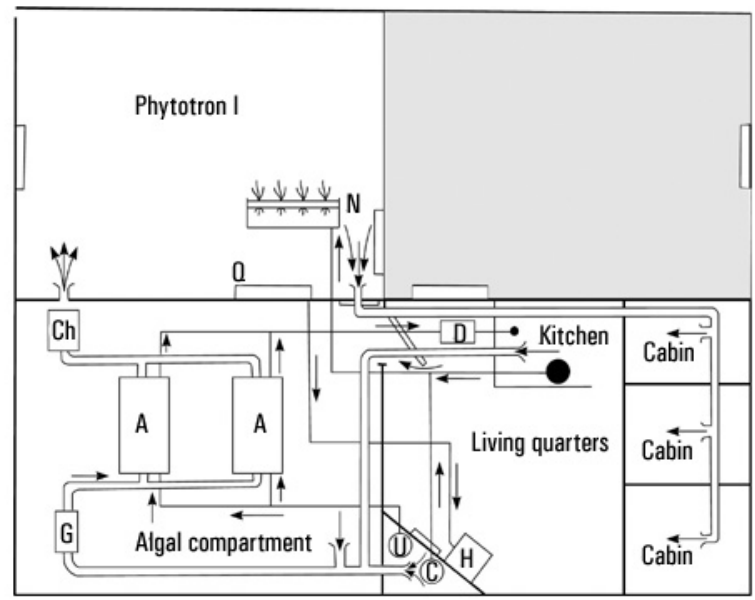

Fig. 4. Schematic of gas and water exchange in the BIOS-3 installation at Phase II of the experiment. A - microalgal cultivator; $\mathrm{G}$ - gas blower; $\mathrm{Ch}$ - charcoal filter; $\mathrm{C}$ - sewage and gray water collection in the toilet and kitchen; $\mathrm{N}$ - wheat nutrient medium; $\mathrm{Q}$ - collector for transpiration moisture condensate in the phytotron; $\mathrm{H}$ - vessel for boiling and storing !hygiene water; $\mathrm{U}$ - urine collector;

D - unit for additional treatment of drinking water by sorption

area with equipment to process wheat and inedible biomass, make repairs and measurements, and purify water and air. One compartment included algal cultivators, which provided enough airrevitalization capacity to support at least three crew members.

If the algal cultivators were removed, each of the three non-crew compartments was used as a phytotron to grow wheat, chufa (sedgenuts), and vegetable crops (Fig. 5). The total growing area was $63 \mathrm{~m}^{2}$, which provided ample air-regeneration capacity.

Each phytotron originally had 20 cylindrical, vertical $6 \mathrm{~kW}$ xenon lamps. By 1991, the number of lamps in one of the three phytotrons was doubled by inserting two lamps into each water jacket. With xenon lamps energized at $220 \mathrm{~V}$, photosynthetic photon flux (PPF) at plant level varies from approximately 900 to $1000 \mu \mathrm{mol} \cdot \mathrm{m}^{-2}$ $\cdot \mathrm{s}^{-1}$ under single lamps and from approximately 1600 to $1850 \mu \mathrm{mol} \cdot \mathrm{m}^{-2} \cdot \mathrm{s}^{-1}$ under double lamps (sunlight can reach approximately $2000 \mu \mathrm{mol} \cdot \mathrm{m}^{-2}$ $\left.\cdot \mathrm{s}^{-1}\right)$.

To maintain the pressure inside Bios- 3 at close to atmospheric levels, which minimizes leaks, two air tanks are connected to the main structure. Air was circulated among the crew quarters and the phytotrons. It was partially purified by the plants, and a thermocatalytic filter; completed the purification by heating the air to 600-650 ${ }^{\circ} \mathrm{C}$, which oxidized organic molecules to carbon dioxide and water (Fig. 6). In the course of the 180-day experiment, concentrations of potentially toxic gases varied around the average values, remaining, on the average, parallel to the time axis, which confirmed that the atmosphere was in a state of dynamic equilibrium. Transpired water was condensed and recirculated, mainly to nutrient solutions for the plants. Some of this water was boiled for washing and general cleaning, but water for drinking was further purified on ion-exchange filters. Small quantities of potassium iodide and fluorides were also added to this drinking water for health, and potassium chloride and some other salts were added to improve the taste (Fig. 7).

The crew communicated with the outside world by phone or through the viewing ports. Samples of various kinds were passed outside through small airlocks for analysis. Electrical signals from sensors attached to the bodies of 


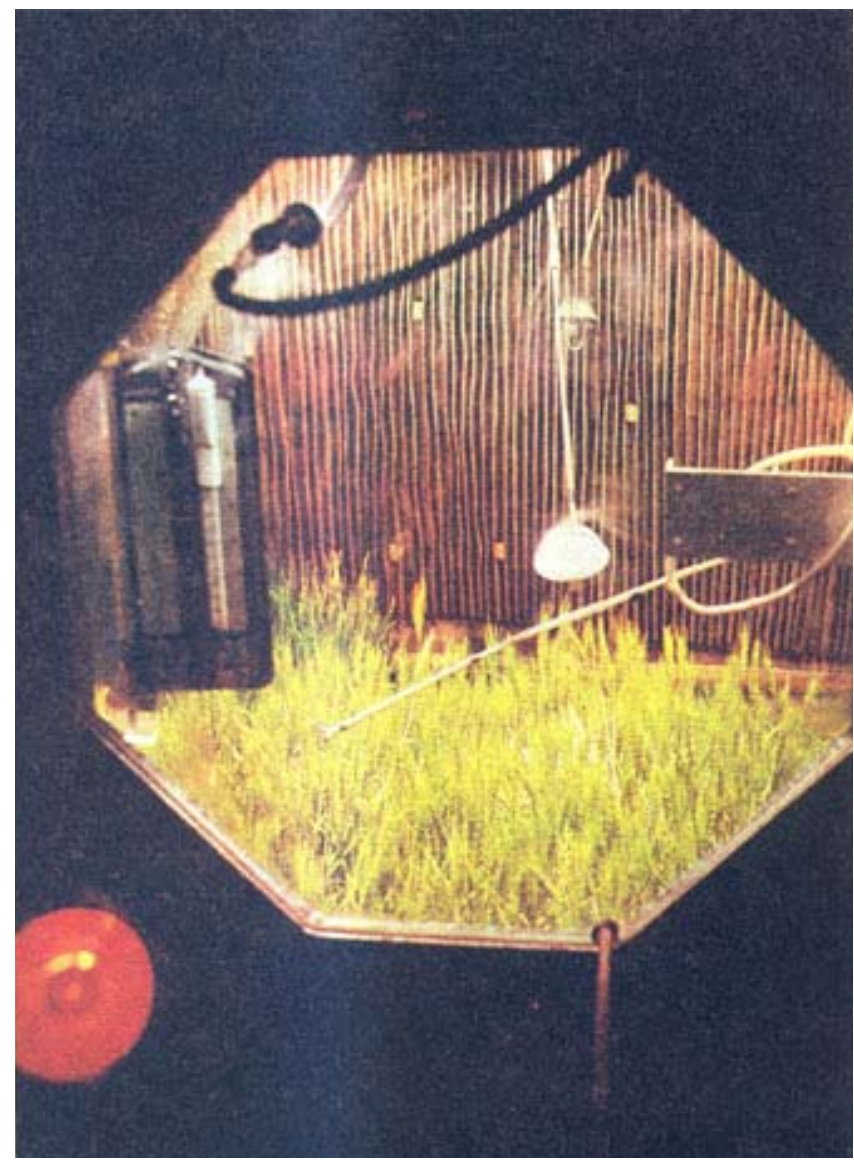

Fig. 5. The phytotron, the view through the porthole

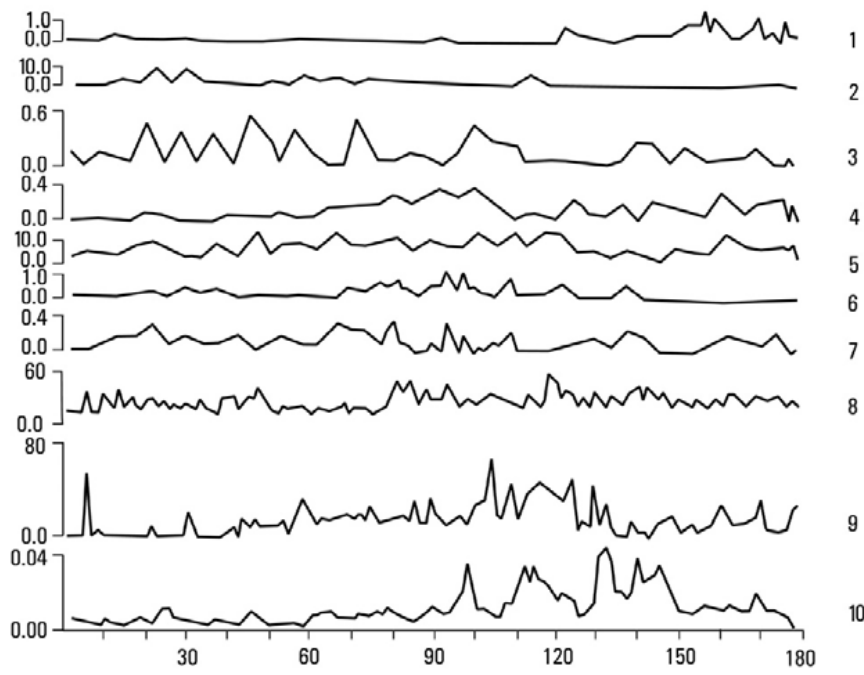

Fig. 6. Changes in potentially toxic gases in atmosphere of a biological system: $1-$ ammonia, $\mathrm{mg} \mathrm{m}^{-3}$; 2 - acetic acid, $\mathrm{mg} \mathrm{m}^{-3} ; 3$ - acrolein, $\mathrm{mg} \mathrm{m} \mathrm{m}^{-3} ; 4$-aldehydes, $\mathrm{mg} \mathrm{m}^{-3} ; 5$-alcohols, $\mathrm{mg} \mathrm{m}^{-3}$;

6 - hydrogen sulfide, $\mathrm{mg} \mathrm{m}^{-3} ; 7$ - mercaptans, $\mathrm{mg} \mathrm{m}^{-3} ; 8$ - organic substances (water soluble), $\mathrm{mg}_{2} \mathrm{~m}^{-3}$; 9 - organic substances (water insoluble), $\mathrm{mg} \mathrm{O}_{2} \mathrm{~m}^{-3} ; 10$ - carbon monoxide, $\mathrm{mg} \mathrm{l}^{-1}$ 


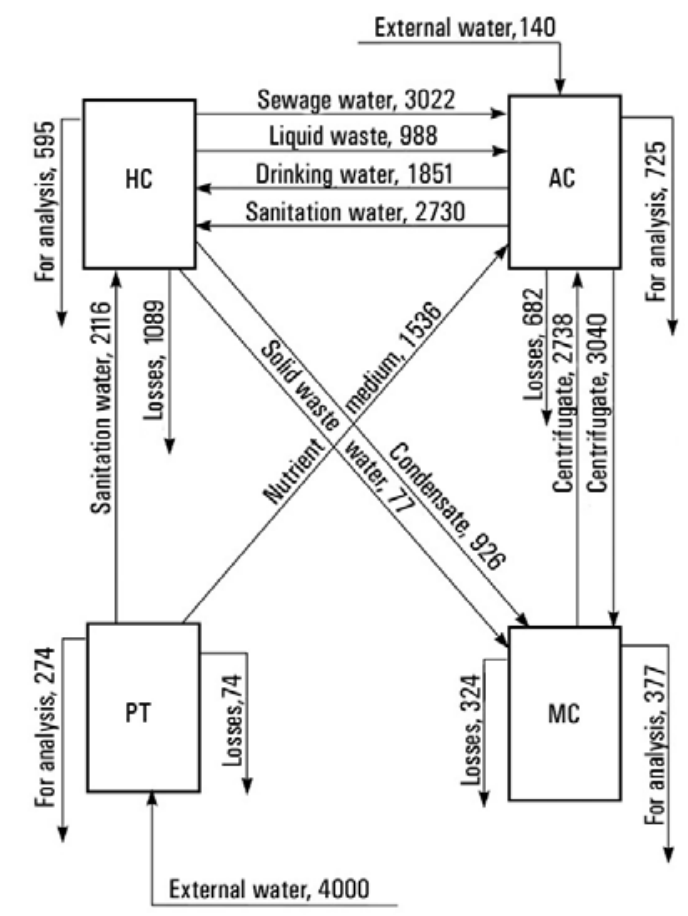

Fig. 7. Schematic of water exchange in the four-link system. HC — cabin with a human; AC - algal cultivator; PT - phytotron; MC - microbial cultivator.

* Figures denote mean daily water expenditure, g.

crew members to monitor various physiological parameters were transmitted to the outside through specially designed sockets.

Bios-3 was the first to make possible experimentation with a long-term human presence in a closed environment regenerated by photosynthetic processes. The atmosphere and water were regenerated completely while food regeneration amounted to $93 \%$ (Table 1). Bios operated for a total of about a year, and continuous experiment with humans in the closed system lasted 6 months (Fig. 8). Some test subjects took part in the experiment 2-3 times; the longest time one human spent inside the system was 13 months (Gitelson et al., 1975, 2003; Salisbury et al., 1997).

Thorough physiological observation involving the measuring of numerous parameters during the experiments and one month after their completion revealed no changes in the test subjects' health condition. After the experiments the test subjects, researchers at the Institute of Biophysics, resumed their research work, presented dissertations, gave birth to children, i.e. lived the lives of ordinary people. Post-experimental observation during 10-15 years showed that none of them had any diseases that could reliably be associated with their stay in the Bios. Hence, we can state with assurance that in the biological closed life support system of the tested design, with biological regeneration of atmosphere, water, and the vegetable fraction of the human diet, the living conditions were adequate to meet the principal human requirements (Gitelson et al., 1989; Kovrov et al., 1985; Lisovsky, 1979). 
Table 1. Daily average yield of biomass in the system phytotrons

\begin{tabular}{l|rr|rrr|rr}
\hline \multirow{2}{*}{ crop } & \multicolumn{2}{|c|}{ area } & \multicolumn{3}{c|}{ edible biomass } & \multicolumn{2}{c}{ total biomass } \\
\cline { 2 - 8 } & $\mathrm{m}^{2}$ & \% of total & fresh, g/day & dry, g/day & \% of total & dry, g/day & \% of total \\
\hline wheat & 17.53 & 44.5 & - & 186.7 & 27.4 & 538.1 & 37.3 \\
chufa & 13.27 & 33.7 & - & 345.6 & 50.7 & 561.8 & 39.0 \\
beet & 0.80 & 2.0 & $93^{\mathrm{a}}$ & 12.6 & 1.8 & 31.3 & 2.2 \\
carrot & 3.55 & 9.0 & 538 & 72.3 & 10.6 & 143.8 & 10.0 \\
radish & 1.94 & 5.0 & 257 & 14.9 & 2.2 & 43.8 & 3.0 \\
onion & 1.00 & 2.5 & 191 & 27.4 & 4.0 & 28.3 & 2.0 \\
dill & - & - & 49 & 5.2 & 0.8 & 5.5 & 0.4 \\
kale & - & - & 51 & 3.9 & 0.6 & 4.0 & 0.3 \\
cucumber & 0.20 & 0.5 & 77 & 3.1 & 0.5 & 8.8 & 0.6 \\
potato & 1.03 & 2.6 & 31 & 4.9 & 0.7 & 71.3 & 4.9 \\
sorrel & 0.08 & 0.2 & 41 & 4.3 & 0.6 & 5.5 & 0.4 \\
total & 39.40 & 100.0 & $1328^{\mathrm{b}}$ & 681.1 & 100.0 & 1442.2 & 100.0 \\
\hline
\end{tabular}

${ }^{a}$ beet edible biomass without tops;

${ }^{\mathrm{b}}$ the total comprises only vegetables.

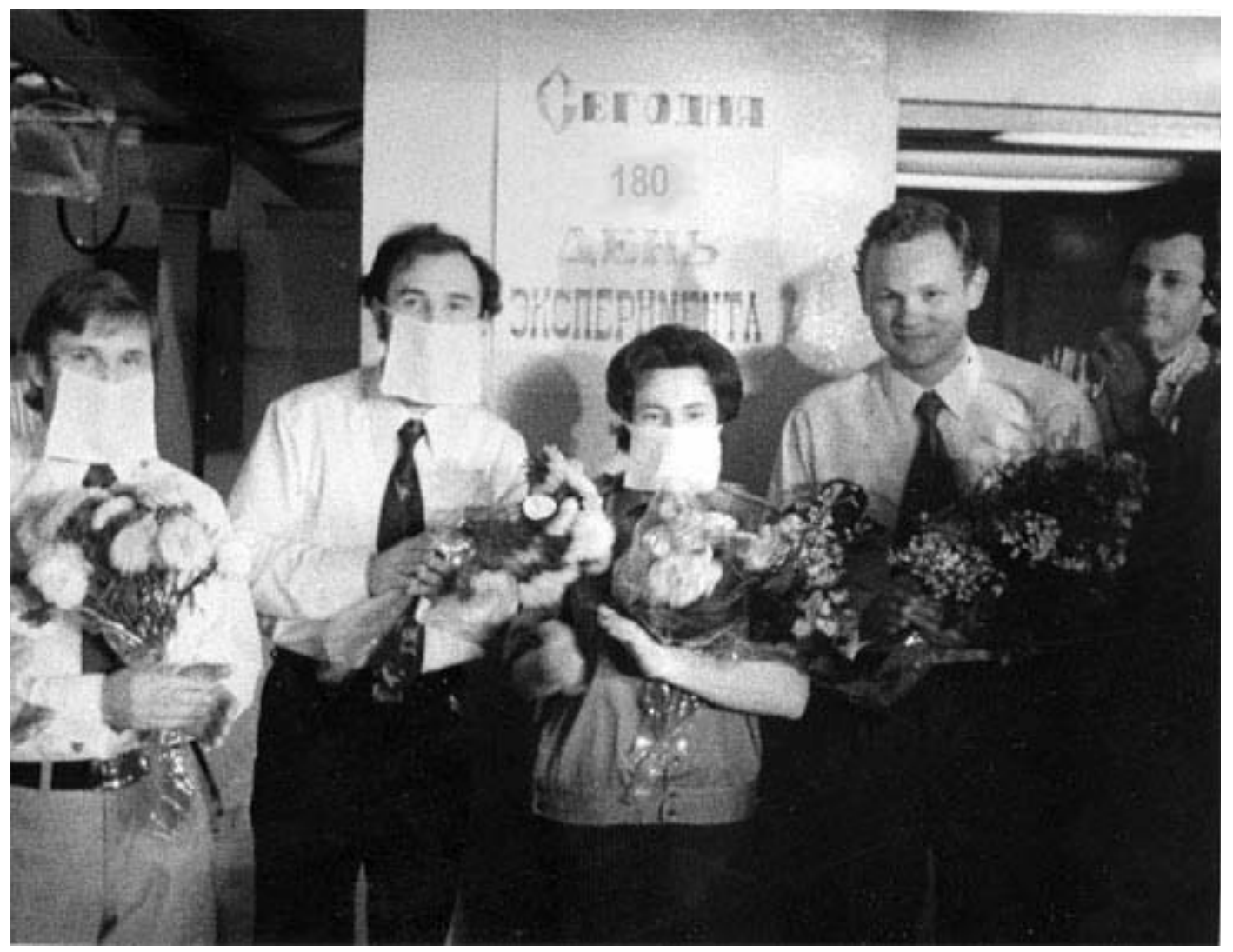

Fig. 8. Three test subjects (wearing respiratory masks) and doctors meeting them when they leave Bios-3 after the 180-day experiment 


\section{The hierarchical structure of ecosystems}

Synthesis of closed ecological systems is a new field of research. It would be well to define the niche occupied by the relatively new branch of ecology, studying the biosphere as a whole.

If ecology is defined as the study of the relationship between organisms and their surroundings, it is naturally subdivided into hierarchic levels differing in the complexity of the ecological system organization:

- elementary ecosystem - relationship of a single individual to its habitat (autoecology);

- population ecosystem-relationship of one-species population to its surroundings (population ecology);

- biocenosis, or community in the American literature - a multispecies ecosystem consisting of many (at least two) species interrelated by trophic, reproductive and signal links (synecology).

A term more commonly used in Russian scientific literature is "biogeocenosis" coined by Sukachev (1944). It emphasizes that a cenosis obligatorily includes not only a living component- "bio"- but also a nonliving one"geo," to which the living component is related through material exchange.

Ecosystems of increasing complexity, from the elementary biocenosis of microorganisms to the multicomponent ecosystems, covering vast territories and water areas, are all at the same level in terms of organization. Their basis is the energy and matter flowing through them.

\section{Biosphere and Noosphere}

The highest level of ecosystems in terms of organization is the biosphere as a whole. The biosphere differs from all ecosystems constituting it by a unique quality - nearly complete closure of the material cycle. So the biosphere is endowed with specific properties justifying detachment of this ecosystem as the object of a separate branch of ecology_biosphere science. In modern literature it is called biospherics although biospherology would be a more logically correct term, while biospherics could only be applied to the part dealing with the synthesis of manmade ecosystems closed to a high degree, the experimental investigation of these systems, and modeling, both physical (natural) and mathematical.

Present-day science and, subsequently, society realize more and more that humanity's technological potential exerts a rapidly increasing effect (mostly destructive) on the closure of the material cycle in the biosphere. Thus, even today the Biosphere experiences a profound influence of humanity, and this influence will grow stronger.

Therefore, there are ample grounds to regard the current epoch in the history of the Biosphere as a transition period of its evolution from the stochastically balanced existence to that controlled by the human race - evolution to the Noosphere (Vernadsky, 1924; Moiseev, 1990; Gitelson et al., 2003).

The emerging branch of science with roots in biospherology but growing into the humanities may be termed noospherology, and the part of it concerned with synthesis of manmade noospheres and their control may be given the name noospherics. In essence, the experimental systems we have described in our artical are just one of the first fruits of noospherics.

Figure 9 is an extremely schematized image of our conception of the relationship between biospherology and noospherology as fundamental sciences, their applied branches - biospherics and noospherics - together with fruits growing on them and buds promising to give fruits in the future.

\section{Humanmade closed ecosystems. Critical, problems and perspectives}

Methodologically this field of science is rather specific since the object itself-closed ecological systems controlled by humans - does not exist in nature. It is likely that the ideal absolutely closed 


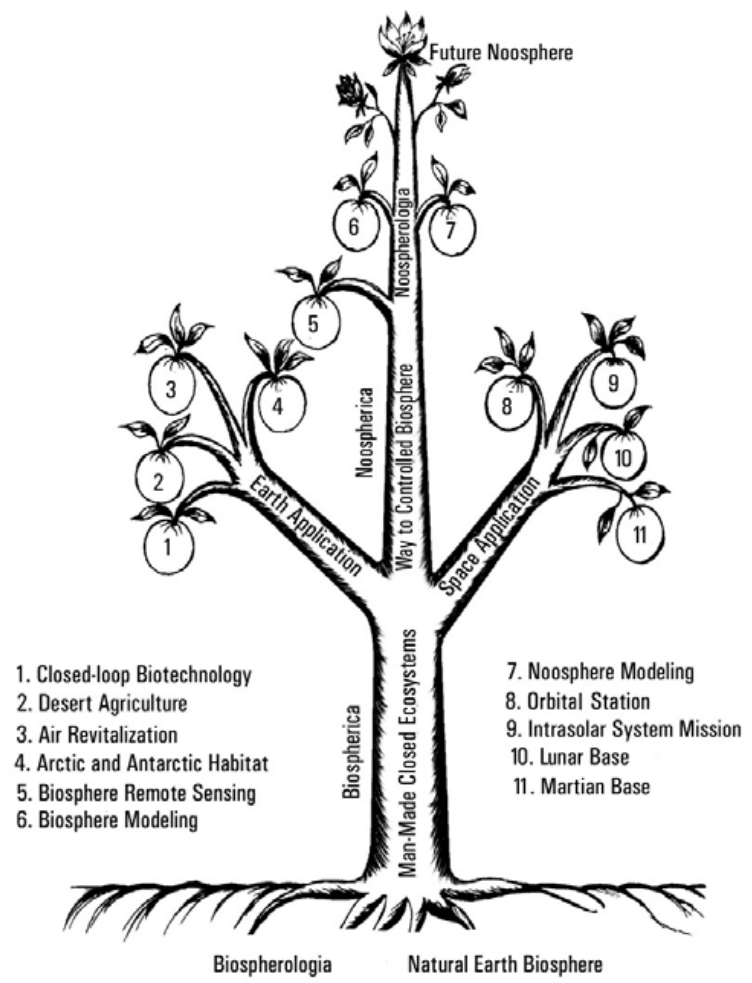

Fig. 9. Tree of prospective Earth and space application of manmade closed ecological system

biological material cycle may be unattainable, but theoretical analysis of the problem shows that this ideal can be approached very closely. Progressing along this path experimentally, researchers, have not as yet encountered any insurmountable obstacles in closing the material exchange in small ecosystems near complete, but not absolutely full closed. In terms of mass exchange, the closure has reached $80-95 \%$, but more closing of the trophic structure is still ahead, and we should expect that each new step will bring increasingly serious difficulties.

Thus, a long distance has been covered on the way to creating closed biosphere-like biological systems within less than half a century. The first "naive" experiments made by Myers (1958) with a mouse under a glass bell, respiring with the oxygen produced by algae, resembles Priestley's classic experiment. The fate of the mouse that died of suffocation in Myers' experiment sadly repeats the history-Priestley's famous experiment. However, as soon as 20 years later people live, breathe and feed for months and months in closed biological life support systems.

Let us, on the basis of the experience that has been accumulated by now, consider the avenues for the further advancement of the described experimental ecosystem directed toward a more complete closure.

Two kinds of obstacles to the further closure of the system and the prolongation of its lifetime can be foreseen: technical and biological obstacles.

\section{Technical or Biological?}

We have named the technical problems first. Although they do not seem fundamental, our experience with the system, particularly with the autonomous version, proves that it is the issue of technical reliability of the system that determines 
to what extent it can be really autonomous. There is a fundamental difference between the live and technical parts of the system with respect to reliability. An organism is a self-sustained system, and a population of organisms is even self-regenerating. Technically speaking, the live part of the biological-technical system not only can be repaired but also can repair itself-it continuously renews itself according to the intrinsic program. This program is the gene pool, and, consequently, the reliability of its restoration depends on the number of autonomously acting genomes. For instance, in the algal reactor of our system it repeats itself in $10^{13}$ autonomous cells. In the case of some damage, one living cell would be enough for the system to restore itself to its original size. One can find experimental support for this statement in (Gitelson and Rodicheva, 1996).

In present-day engineering, the functions of operation and restoration are performed by different devices. Machines cannot restore themselves, and their survival is provided either by redundancy or by maintainability. That is why in the biological-technical system, where live and technical parts are as close as organs in a single organism, the overall lifetime is determined by the most short-lived element - and that is the technical part. For many years we have been dealing with experimental closed systems and do not have a single record of system failure for biological reasons. All damages were the fault of technical devices.

A biologist-reader may argue that bacteria, insects, or other organisms that multiply at a high rate can quickly increase their numbers in the ecosystem and cause a catastrophe. This possibility cannot be excluded, a priori. However, no new species can invade the closed system, just because it is closed, and the species inhabiting it are at equilibrium, limiting each other. Mutants can certainly emerge there; moreover, the rate of mutation must be the same in the closed system and in the natural environment, but a mutant would hardly find its eco-niche in the closed system, where the conditions remain unchanged and under control. For many years we have been conducting experiments in closed ecosystems, but have not dealt with any spontaneous biological catastrophe. General biology and the evolution theory would benefit from an investigation of the possibility and probability of such biological catastrophes in closed ecosystems. This can be done using the material of high statistical power: hermetically sealed microcosms, closed ecosystems consisting of unicellular organisms.

The second technical aspect is the scheme of controlling a small closed ecosystem. Manmade closed ecosystems differ from their prototype, the biosphere, by the principle of control. The sustainability of the biosphere is ensured by its biological diversity creating an intricate network of metabolic paths with fail-safe redundant functions, by buffer stocks of bio-inert mass, and by the huge size of the planet itself. Such a system, produced and polished by evolution, is sustainable due to stochastic control. By contrast, in a small manmade closed ecosystem all these factors become ineffective: its diversity and size are not sufficient for stochastic mechanisms to operate successfully; it also lacks history, during which stabilizing selection could take effect.

A deterministic control system is a prerequisite of the sustainable existence of a small, closed, ecological system. If we look for a parallel in living nature, the closest will be the central nervous system, controlling a multicellular organism. The human introduces into the manmade ecosystem a regulation system and a scheme of control. This is the fundamental difference between humanmade ecosystems and any natural ecosystem, and this is also a condition of its sustainability. 
Human intellect must be involved not only in creating a small closed ecosystem but also in maintaining it in a sustainable state. That is why we have to recognize that by the principle of control manmade ecosystems are rather models of the noosphere, the future state of the natural biosphere.

The main result of our experiments is proof that an internally-governed biological life support system can be realized and maintained in a sustainable state within the bounds of a tightly limited space on the condition that it is obligatorily controlled by the human intellect - " noos".

A problem arises of devising equipment whose vitality would correspond to the reliability of the biological part of the system. An ideal solution would be to invent self-repairable devices by analogy with living organisms, but this can hardly be the destiny of the nearest generations of devices. Now it must be concluded that of the two parts of the biological-technical union it is the technical part that is unreliable, and this factor will limit the lifetime of autonomous life support systems. We are emphasizing this conclusion because it is in conflict with the opinion popular among engineers, that machinery is more reliable than a fragile and unreliable living body.

Here it would be appropriate to sum up a long discussion between the proponents of physicochemical and biological life support systems. Experience shows that their development is convergent - the future is with hybrid biological-physicochemical systems. However, this hybrid cannot be a mere summation of similar functions of the current systems.

It is our opinion that in the hybrid system the main function of the biological link will be to provide humans with regenerated products, while the physicochemical link will maintain the operation of the biological link and recycle dead end products of the live part of the ecosystem.

\section{Food Problems}

Let us look at the problems of the biological part of the closed ecosystem with the human. Two principal problems stand out here. First, it is further closing of the system trophic structure. The main problem is to match the food inlet of the human link and the biomass regenerated within the system. The difficulties here are twofold. To begin with, it has not been clearly defined yet what is an "optimal human diet," whether we know all the compounds required by a human, their quantities and proportions, whether they can replace each other, and what the minimum required diversity is. It is highly probable that the so-called balanced diet is not optimal to follow for a long time as it can result in the detraining of adaptation mechanisms. However, the most important question is if the traditional sources of human food are irreplaceable. There are ample grounds to think that the range of food animals and plants, which is narrow compared to the species diversity of the biosphere, has been selected by humans in the course of history rather for economic and technological reasons than for the unique biochemical properties of these food sources. Acknowledgement of this idea opens up a lot of opportunities for detecting new food sources among animals and plants, more fit to be intensively cultivated in a manmade ecosystem than traditional agricultural objects (Kamarei at al., 1986; Salisbury at al., 1995).

On the other hand, our reasoning has to be based on the fact that the human diet, which has formed empirically, is adequate for people's requirements. This is one of the physiological and psychological stereotypes that must be approached extremely carefully, bearing in mind a lag in the organism's response to changes in diet.

The second aspect of the food problem is the impossibility of selecting one species, a "metabolic antipode" to the human, that would 
regenerate all the food required by the human, feeding all human excreta.

We have to cultivate in the system multispecies biocenoses, which complicates its structure. The size and energetics of the system are particularly affected by the augmentation of trophic levels. To synthesize $50 \mathrm{~g}$ of animal protein a day, the phototrophic link must increase twice, i.e. the expenditure of the system is equal to that needed to produce the other $500 \mathrm{~g}$ of the primary biomass for the human. Naturally, researchers strive to make the system trophic structure as simple as possible and, first of all, to base the regeneration of all food for the human on the autotrophic processes of primary biosynthesis. Agricultural plants perform this function fairly well, regenerating carbohydrates, vegetable oils, and proteins. However, up to half or more of the biomass synthesized by plants is inedible, being a deadlock product. Ways of returning it to the material cycling are yet to be found.

A temptingly easy solution to the problem for space life support systems and for the expanding Earth's population would be to turn to a vegetarian diet, to give up eating animal food. The efficiency of agricultural production would at least double; and the possibility of living on a vegetarian diet has been proved by the experience of many people.

In our opinion, however, this cannot be recognized as an optimal way to solve the food problem either in space or on Earth, since it is founded on a considerable limitation of human physiological and psychological requirements for most people, who are not vegetarians.

Modern science is able to propose another way-to use biotechnological methods in synthesizing an absolute biochemical equivalent to the normal human diet, preserving the organoleptic properties people are used to.

The production of animal protein or its biochemical equivalent remains the most difficult problem. In terms of amino acid composition, $300-400 \mathrm{~g}$ of algal biomass contain sufficient amounts of all essential amino acids, including the most scarce - sulfur-containing — amino acids. In this case, however, the diet would include excess quantities of protein, to $150-200 \mathrm{~g}$, nucleic acids, to $15-20 \mathrm{~g}$, excessive pigments, potassium, magnesium, and sulfur. Similar products can be obtained by cultivating hydrogen-reducing or other chemoautotrophic bacteria.

By controlling the direction of biosynthesis parametrically, it is feasible to bring the composition of the produced biomass much closer to human food requirements. To illustrate, the protein content of Chlorella can be reduced more than twice, to 20-25\%; amounts of nucleic acids, chlorophyll, and mineral substances can be also decreased. However, a gap between human requirements and the products of algal biosynthesis is still wide, and attempts to make it narrower lead to the reduced productivity of photosynthesis.

Another way is the physicochemical transformation of the primary biomass or extraction of parts that can be used for food. Development of this line has started only recently, but it may be expected that a large part of the synthesized biomass can become edible. This is the most realistic approach used today.

Bright prospects are held out by genetic engineering and selection, ways to control the program of biosynthesis so as to attain the maximum correlation between the composition of the primary produced biomass and human food requirements. The closed ecosystem offers favorable conditions for selection-genetic work. The feasibility of having 5-6 generations of wheat a year or autoselection processes going on in microalgal and bacterial continuous cultures are the factors that can permit significant directed shifts in the programs of their biosynthesis to be attained within reasonable time. 
Genetic engineering offers prospects for constructing a genome of the organism that would be an absolute metabolic opposite to the human. Hopefully, it would be possible to create such a controlled system with the human that would in essence make the "human plus a genetically modified plant" complex as an autotroph system, exchanging only energy with the surroundings. However, this is still a matter for the distant future.

Limiting ourselves to the prospects attainable by currently available methods, we can state that using parametric and selection-genetic methods of controlling the direction of biosynthesis together with the physicochemical processing of the products of biosynthesis may bring us much nearer to the complete closure of the human food cycle in the system.

The second objective that became evident in experiments with the ecosystem is the maintenance of its biological stability. Besides the technical issues discussed above, two biological problems revealed themselves - trace elements and concomitant potential viruses and bacteria.

\section{Trace elements problem}

The migration of trace elements in the experimental system has turned out to be a hardly controllable process, as trace elements enter into the material cycle from system construction materials, are captured and multiply concentrated by system inhabitants in a different way depending on the body part, on the stage of life, and on the state of the organism. Note that this is not an engineering problem even though trace elements are supplied by the system's construction materials. They are not released by passive erosion, but rather due to the diverse biochemical activities of the microflora exhibiting versatile aggressiveness. Further migration of trace elements involved in element cycling is also determined by biological processes in the system. This threat to system balance was revealed in experiments with closed systems. To find ways of controlling the dynamics of trace elements in closed ecosystems is an important objective of future investigations.

We can show the importance of microflora as a possible source of system disturbance using its contribution to the mobilization of trace elements as an example. The rich gene pool of concomitant microflora constitutes a potential threat that new, unplanned processes would take place in the closed ecosystem.

\section{Microflora in CELSS}

The dynamics of the microflora in the ecosystem during the experiment not reach an absolutely steady state. This was true for human flora in particular. This proves that the system's equilibrium can be really upset by bacterial processes. Viruses may also function as a genetic nuisance, though we had no chance to see any evidence of their activity in the system. To find ways of controlling the state of concomitant microflora still remains an urgent task (Gitelson et al., 1980).

The position of microflora in the ecosystem is different from the natural situation in several ways. We would like to place special emphasis on some conclusions.

Neither the closure of the experimental ecosystem, nor its small size, nor its bacteriological isolation from the outer environment, nor the intensification of its metabolism under the conditions of extremely simplified trophic structure, nor the large flows of microflora from one link to another caused uncontrollable, destructivefor-the-system outbreaks of microorganisms, nor dangerous changes in their species composition or metabolic properties. It seems that in the small closed ecosystem, at least in the versions we have investigated experimentally, the mechanisms ensuring the stability of the microbial biota 
remain efficient. This renders us optimistic about the sustainability of closed life support systems.

The four-month experiment with the system "human-higherplants"wasconductedinthesystem protected from the invasion of microorganisms from the outside, by excessive internal pressure. The experiment has demonstrated that the system microflora remains essentially the same. Hence, the conditions and capacity of the system are adequate for microflora to sustain itself without any contact with the outer environment.

Another significant regularity of this manmade ecosystem is that despite intensive exchange between links no shifts in their microflora occur, and microbial landscapes remain specific for each link. Thus, the ecological factor remains the dominant one in controlling microflora in the manmade ecosystem. This circumstance is essential for substantiating the choice of the method for controlling microbiological conditions in the closed ecosystem. The choice is either to establish sterile intra-system barriers or to allow nearly free exchange limited only by hygienic measures precluding the seeding of living quarters with fungal spores and bacteria that may be hazardous to humans.

The results of the experiments argue for the ecological method of microflora control, i.e. for controlling the composition and numbers of microflora by maintaining proper environmental conditions. The alternative method-the establishment of sterile barriers between ecosystem links - is difficult to realize technically in a life support system of limited size and possibilities. Sterile barriers may also be more dangerous than an ecological balance: if the barrier is broken by accident, which can hardly be avoided, the microflora, which has been developing in isolation until this moment, can change quickly and profoundly (Somova, 1996).

\section{Experimental humanmade CES}

Until now we have discussed an experimental closed system with human inhabitants. The demand for such systems has been created by the advance of cosmonautics, but the results of the work can be applied in different spheres, and the ecosystems for such applications need not necessarily include humans.

Investigations of the closed ecosystem may be of theoretical significance because it is a fundamentally new formation, possessing the properties of a biocenosis in terms of species composition, the properties of the organism in terms of the principle of control, and, finally, an absolutely unique property of exchange closure, which has no analog in nature, except for the biosphere as a whole. This object is exceptionally interesting for studying some fundamental properties of living systems. The closed ecosystem, a microworld created by the experimenter, offers an opportunity to reveal the regularities of the existence of closed ecological systems as models of the Earth's biosphere, to define the limits of sustainability, and to observe the transition processes of restoration after the system has been disturbed. The rate of cycling in these ecosystems can be thousands of times higher than in the biosphere. This affords new opportunities for experimental ecology and physiology, for modeling genetic-population and evolutionary processes in the small biosphere, every part of which is transparent, visible, and accessible for analysis and interference. The introduction of the human into the closed ecosystem makes it possible to reveal all human metabolic relations with the environment, including vanishingly small ones, the effect of which can be seen only if the cycles are repeated many times. These opportunities must be primarily used to investigate the physiology and biochemistry of the exometabolism of both normal and sick humans and, probably, to subject them 
to curative treatment. Those are some research opportunities of experimental ecosystems.

However, in spite of the importance of the objectives, only six closed biological human life support systems have been created by now or are being created: two in Russia - the Ground Experimental Complex in Moscow and the Bios in Krasnoyarsk (Allen, 1993); and two in USA: Biosphere2 and in the NASA Johnson CenterBIO-Plex (Tri et al., 1996); one in Europe (SpainMELISSA, ESA) (Melissa, 1998); and one in Japan (CEEF) (Nitta et al., 1996). It would not make sense to construct more of such systems in the near future as they are all very costly, experiments with them are complicated, and information is accumulated slowly. It may be more expeditious to develop miniature closed ecosystems-miniCES. The International Center for Investigations of Closed Ecological Systems of the Institute of Biophysics has devised such a desk-top CES, a one-tenth scale model of the full-sized CES with a human, but also including the human metabolism. The rate of data accumulation in the CES depends more on the number of material cycles in it than on the length of the period of operation in terms of solar time. Therefore, such a mini-system as a research tool is as many times more efficient than a full-scale system as the rate of the cycle in it is higher than the rate of the cycle in the full-scaled system. The cost of manufacturing and operating such a system is tens, or even hundreds, of times lower than that of the full-scale system. It can be easily cloned and used as a research tool in many laboratories. In the present-day situation, when it is not only Russian science that lacks material support, this is of great importance.

It would be reasonable to keep full-scale CES as unique centers for periodic, probably not very frequent, key experiments that would demand the presence of humans in the system. The world-wide significance of such experiments and their high cost make it imperative that centers possessing such experimental systems should be officially recognized as open laboratories - international centers. The Center for Investigations of Closed Ecosystems of the Institute of Biophysics, Siberian Branch, Russian Academy of Sciences, in Krasnoyarsk, has the status of an international center and is open to scientists from all over the world.

Quarantine measures on the planets and other celestial bodies to be visited by humans can be better provided for by closed life support systems than by open ones. The closed ecosystems will not release into the outer environment any living material, particularly microorganisms, capable of autocatalytic colonization of space, and, therefore, will not disturb the intact state of the planet. This is the way to protect nature from destructive consequences, which often accompanied the expansion of civilization in the past.

A warning from the past can be provided by the example of Europeans visiting the isolated islands of Oceania and introducing infections against which the aborigines had no immunity and, therefore, died. Of course, we cannot seriously expect a meeting with anthropomorphous inhabitants of other worlds, but even microbial contamination of the celestial bodies could be rather dangerous and, anyway, undesirable.

This objective of cosmonautics can be defined as the creation of a system optimally providing the human requirements with minimum expenditures and without environmental pollution. But isn't it the most vital current problem of humanity on Earth? The similarity of these problems lends great importance to the creation of manmade ecological systems for humans.

\section{Terrestrial applications}

It seems unlikely that anyone can predict now when we are going to need closed life support systems outside Earth, but they can find terrestrial applications even now. 
For people living in the most severe conditions on our planet it may be more reasonable to design essentially closed ecosystems and maintain the optimal environment inside them than to condition the environment in the open habitat. For northern settlements systems of cascade energy use can be of interest.

According to the project of ecological habitation for northern territories devised at the Institute of Biophysics, an energy cascade with an initial power of $12 \mathrm{~kW}$ could provide for a house inhabited by a family of $5-7$ members with lighting and heating, as well as supply people during the northern winter and even polar night with fresh vegetables and berries; it will also absorb human wastes without polluting the environment (Bartsev et al.. 1996).

Another promising application of closed ecosystems is related to the Sick Building Syndrome (SBS). This is a serious problem not only for such completely sealed products of high technology as spacecrafts and submarines but also for considerably less sealed living and working spaces, where people inhabiting medium and northern latitudes spend the major part of their lives. The problem has unexpectedly become much more acute after the energy crisis of the 1970s, caused by irregular supplies of oil. In an attempt to reduce the energy expenditures for the heating and conditioning of buildings, additional sealing was done. The better it was performed technically, the higher was the probability of SBS in the inhabitants of these buildings. A flow of papers dealing with the SBS problem ensued, but the mechanism of its origination has not been determined exactly. Against this background the results of our experiments in the Bios-3, which revealed no signs of SBS in our test subjects who stayed for months in the sealed space regenerated by plants, are very important and can be used to overcome SBS in modern highly sealed energyefficient buildings (Wolverton et al., 1984).

\section{Predications and confirmation}

Having covered some distance, it may be well to remember the start.

In the Chapter "Ecology of Spaceflight" written by G. Cooke ${ }^{2}$ for the famous book "Fundamentals of Ecology" by E. Odum, published several times in the 1970s (Odum, 1971), the author analyzes the prospects of creating biological life support systems, which at that time were still to be devised. G. Cooke pinpointed the difficulties and obstacles to be overcome on the way to creating a biological system that regenerates the environment for a human. He based his opinion on his experience as an ecologist well aware of the properties of open, non-closed ecological systems.

We will quote G. Cooke here and see if his predictions have come true. Let us compare Cooke's forecasts with later experimental results.

1. The system with microalgae is difficult to control. The experiment made by Eley and Myers (Eley, Myers, 1964) with the gas-closed system "algae-mouse" that lasted 82 days is one of the longest investigations of this kind. Basing on ecological theory, we can predict that it will be extremely hard to stabilize such systems. Therefore, complicated outer mechanisms are needed to ensure quite a steady level of nutrients.

It seems highly unlikely that a system including only one dominant species, a producer, could be maintained in a steady state for a long time unless huge amounts of energy are expended on regulation. For the system to be practicable the regulators must be extraordinarily large and complicated.

As has been shown in our long-duration experiments, the system is reliable and rather

Quotations from G. Cooke are taken from the Russian version of E. Odum's "Fundamentals of Ecology". 
simple to control. Control takes not more than a quarter of the energy provided by photosynthesis.

The reason is that intraorganismal processes form a steadily autocorrelated metabolic network, and if, measuring them, we can make the right choice of one parameter to be monitored, we will be able to control the whole system. For a microalgal population, this parameter is the growth rate, which can easily be measured continuously by an increase in the optical density. Knowing the correlation between the biosynthesis rate and the requirement for biogenic elements, measurements of growth rate will suffice to maintain steady-state conditions for algal cells in continuous culture for an indefinitely long time.

Another important factor of control is the closure of the system. The two links of the system — human and microalgae — consume each other's gaseous exometabolites, exchanging $\mathrm{O}_{2}$ and $\mathrm{CO}_{2}$ in respiration and photosynthesis, and thus controlling the photosynthetic rate by limiting $\mathrm{CO}_{2}$ inflow. So, a feedback system is established between the human and microalgae. Under the conditions of a closed gas cycle, microalgal productivity is regulated through this system (Myers, 1958).

This method of regulation has been verified in our experiments for many years, in which thousands of microalgal generations succeeded each other in continuous culture, providing humans with air for respiration and showing no signs of inhibition or degeneration (Fig. 10).

\section{Algal culture is unreliable. In experiments}

on creating systems 'algae-mammals' many complications arise: invasions of competitors or predators (bacteria or zooplankton), build-up of algal toxins, emergence of algal mutants with decreased levels of metabolism. These problems were all to be expected in an oversimplified ecosystem without adequate internal regulation.

We recorded none of the predicted phenomena. In our opinion, the reason is that in our experiments we did not deal with axenic culture. It was a biocenosis with symbiotic relations between the algae and the bacteria.

As for predators and competitors, there is no way that they can be introduced into the closed system. Finally, the main complication predictedspontaneous emergence of slowly growing mutants - has been combated by continuous turbidostat cultivation. Under such conditions, similar to natural selection in essence, quickgrowing cells and their progeny have a higher chance to survive than "slow" mutants, which will be naturally washed out of the cultivator, having no chance to multiply and become dominant in the cultivator. Darwinian selection is inexorable. Experiments (Gitelson and Rodicheva, 1996) (Fig. 11) demonstrate the efficient action of this mechanism in the event of UV-injured Chlorella.

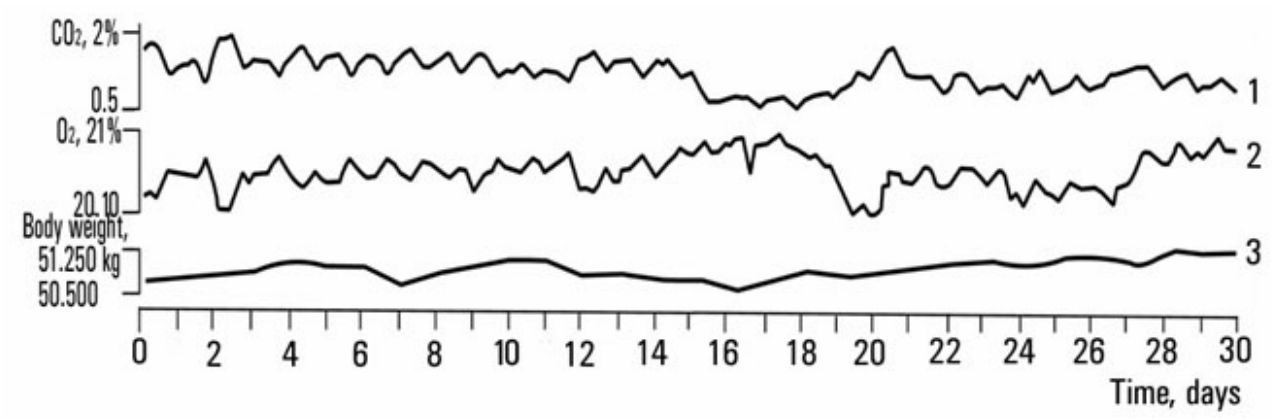

Fig. 10. Dynamics of the carbon dioxide and oxygen concentration and the weight of a man under observation in a 30-day experiment. $1-\mathrm{CO}_{2}$ concentration; 2 - absolute $\mathrm{O}_{2}$ concentration; 3 - body mass 


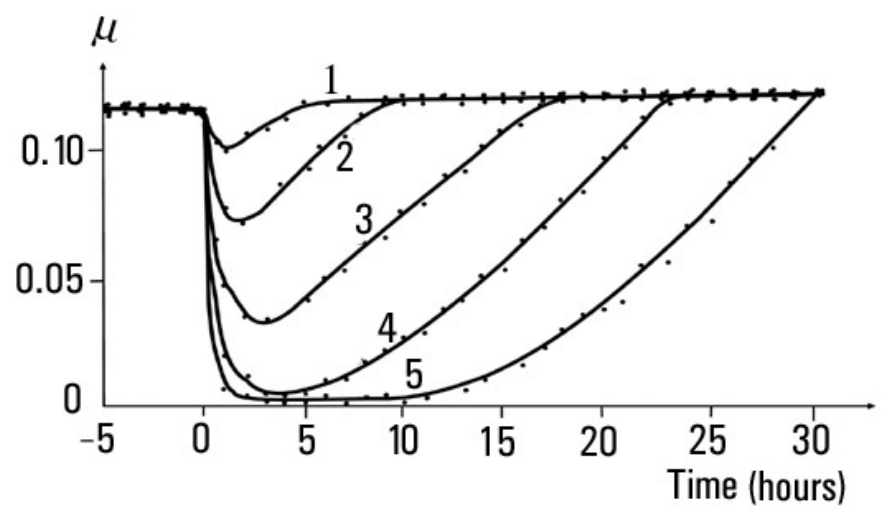

Fig. 11. Dynamics of specific growth rate changes (h-1) for a culture exposed to different UV doses. $1-2.8 \cdot 10^{2} \mathrm{~J} \mathrm{~g}^{-1} ; 2-5.6 \cdot 10^{2} \mathrm{~J} \mathrm{~g}^{-1} ; 3-11.2 \cdot 10^{2} \mathrm{~J} \mathrm{~g}^{-1} ; 4-16.8 \mathrm{~J} \mathrm{~g}^{-1} ; 5-22.4 \cdot 10^{2} \mathrm{~J} \mathrm{~g}^{-1}$

Even if for some unpredictable biological or (much more likely) technical reasons the whole algal population does die, only a phenotype will disappear. It is not very difficult to restore the population by the conserved genotype. The necessary information is built into each Chlorella cell (or any other organism) measuring only $30 \mu \mathrm{m}$. To be on the safe side, the gene pool can be stored in many replications.

3. Human-algal gas exchange cannot be steadily balanced due to a difference between $R Q$ and $A Q$.

It has been shown in our experiments (Okladnikov et al. 1979), that a balance between algal AQ and human RQ is achievable on the basis of the steady controllable growth of algae on a balanced nutrient medium and the correction of the human diet.

In conclusion it should be said that G. Cooke's predictions advanced a priori were quite legitimate and founded on the thorough ecological analysis of the situation, based on the knowledge available by that time. This knowledge led him to the pessimistic conclusion that use of such simple ecosystems imposes severe hazards on the astronaut, and their development as the basis of a life support system should be discontinued.

Nevertheless, we did not give up this work almost 40 years ago, and now, a posteriori, we see that these forecasts have not come true. By investigating the functioning of such systems, we have verified their reliability experimentally and, what is important, we know which stabilising biological mechanisms act in these systems. Our conclusion, supported by this knowledge and its experimental verification, is the opposite to $G$. Cooke's conclusion, and it is quite optimistic: the creation of closed ecological human life support systems, beginning with the simplest one-with human-microalgal gas exchange, is feasible, and they are at least as reliable as physicochemical systems performing the same function. And they have already been tested. Methods of control developed for them proved to be applicable to higher-plant cultivation. On this basis we devised mulispecies systems, such as Bios-3, which can be efficiently controlled without enormous expenditures. This opens up possibilities for their space applications. Our confidence has been essentially supported by the results of recent experiments on the Mir station performed by the joint Russian-American research team. They have shown that plants (wheat, at least, which is most important) can grow almost normally in microgravity, if the technologically controlled environment is suitable (Salisbury et al., 1995). 


\section{Conclusion}

However, neither future space flights nor the potentials of terrestrial applications of manmade closed ecosystems seem to us so significant as their application as teaching aids demonstrating the vital importance for humans of preserving the biosphere.

At the same time, the last thing the authors want is that their warnings about the destiny of the biosphere could be understood by readers as unrestrained "green" alarmism. On the contrary, we see the way out of the collision between humanity and the biosphere, which has produced it, in using scientifically substantiated methods for controlling the biosphere. An unchecked advance of scientific knowledge in the twentieth century gives us reasons to hope that the twenty-first century can become the period of harmonization of relations between humans and the biosphere. However, this can be only attained on the condition that humanity would realize soon enough the inadmissibility of further wastage of huge material and intellectual resources for the satisfaction of political ambitions, for national clashes, for wars between each other and with nature. Only a coordinated strategy of interaction with nature, pursued by humanity as a whole, can ensure the transition from the impending danger of the chaotic exploitation of the biosphere by humans to a controlled Noosphere. It is hardly possible to find a more vital and universal objective of presentday science than to comprehend the mechanism of the everlasting existence of the biosphere and to propose to society a scientifically substantiated strategy for controlling the biosphere with the aim of preserving this mechanism. This knowledge must become the foundation of our outlook on the world for the generations to come.

The people responsible for strategic political decisions must realize that now, in the twenty-first century, we are in a fundamentally new situation: relations between humanity as a whole and the biosphere rather than human interrelations are becoming critical for the future of both Humanity and the Biosphere.

\section{References}

Allen J.P. (1993) Using a closed ecological system to study Earth's biosphere. Bioscience 43: 225-236.

Bartsev S.I., Mezhevikin V.V., Okhonin V.A. (1996) Bios-4 as an embodiment of CELSS development conception. Advances in Space Research 18: 201-204.

Gitelson J.I., Kovrov B.G., Lisovsky G.M., Okladnikov Y.N., Rerberg M.S., Sidko F.Y., Terskov I.A. (1975) Problems of Space Biology. Vol. 28. Experimental ecological systems including man. Nauka, Moscow, 311 p. (in Russian)

Gitelson J.I., Manukovsky N.S., Pankova I.M., Somova L.A., Tirranen L.S. (1980) Microbiological problems of closed ecological systems. Nauka, Novosibirsk, 198 p. (in Russian).

Gitelson J.I., Lisovsky G.M., Kovrov B.G., Okladnikov Yu.N. (1989). Long-term experiments on man's stay in biological life-support system. Advances in Space Research 9: 65-71.

Gitelson J.I., Rodicheva E.K. (1996) Self-restoration a specific feature of biological life support system. In: SAE Technical Paper Series 961495. 26th International Conference on Environmental Systems; 1993; Monterey, CA. 8-11. Available from: SAE, 400, Commowealth Dr., Warrendale, PA 14096-0001. 
Gitelson J.I., Lisovsky G.M., MacElroy R. (2003) Manmade Closed Ecological Systems. Taylor \& Francis, London,400 p.

Kamarei A.R., Nakhost Z., Karel M. (1986) Potential for utilization of algal biomass for components of the diet in CELSS. In: MacElroy R.D., Marrello N.V., Smernoff D.T. (eds), Controlled ecological life support systems: CELSS 85 Workshop. p. 13-22 (Available from NASA Ames Research Center, Moffett Field, CA 94035, Technical Memorandum TM88215)

Kovrov B.G., Terskov I.A., Gitelson J.I., Lisovsky G.M., Okladnikov Yu.N. (1985) Artificial closed ecosystems "man-plants" with a full regeneration of atmosphere, water, and ration vegetable. Proceedings of the XXXVI International Astronomy Congress; 7-12. Oct 1985; Stockholm, Sweden. Pergamon Press, New York.

Lisovsky G.M. (ed., 1979) Closed system: man-higher plants (in Russia). Novosibirsk (Russia): Nauka Press. [Available in English as a NASA Technical Translation,1981].

Melissa (1998). Final report (1997) activity ESA/EWP. Lasser Ch. (ed.), 113 p.

Moiseev N.N. (1990). Chelovek i Noosphera (Human and Noosphere). Molodaya gvardia, Moscow. 434 p. (in Russian).

Myers J.I. (1958) Study of photosynthetic regenerative systems on green algae SAM. Randolph AEB Texas USAF School of Aviation Medicine Report. 58: 117.

Nitta K., Ashida A., Orsubo R. (1996) Closed ecological experimental facilities. Life Support and Biosphere Science 3: 101-115.

Odum E. (1971) Fundamentals of Ecology, 3-th Edition, Chapter 20. W.B. Saunders Company, Philadelphia, London, Tokio, p. 629-643.

Okladnikov Y.N., Vlasova N.V., Kasaeva G.E., Rodionov V.N. (1979) Human link in experimental (medical-physiological investigations). In: Lisovsky G. (ed.) Closed system human - higher plants. Nauka, Novosibirsk, p.82-99. (in Russian)

Salisbury F.B., Bingham G.E., Campbell W.F., Carman J.G., Bubenheim D.L., Yendler B., Jahns G. (1995) Growing super-dwarf wheat in light on Mir. Life Support and Biosphere Science 2: 31-39.

Salisbury F., Gitelson J., Lisovsky G. (1997) Bios-3: Siberian Experiments in Bioregenerative. Life Support and Bioscience 47: 575-584.

Somova L.A. (1996) Effect of closure on man-microbes interactions in different types of Earthspace missions. 31st Scientific Assembly of the Committee on Space Research (COSPAR); 14-21 July 1996; Birmingham.

Sukachev V.N. (1944) On the principles of genetic classification in biogeocenology. Zhurnal Obshchei Biologii. 5: 213-227 (in Russian).

Tri T.O., Edeen M.A., Henniger D.L. (1996) Advanced life support human-rates test facility: testbed development and resting to understand evolution to regenerative life support. SAE Technical Paper Series nr 961592. 26th International Conference on Enviromental Systems; 8-11 July 1996; Monterey, CA. Available from SAE, 400 Commonwealth Dr., Warrendale, PA 14096-0001.

Tsiolkovsky K.E. (1926) Exploration of world space with rockets. Kaluga, 83 p. (in Russian)

Tsiolkovsky K.E. (1964) Life in interstellar medium. Nauka, Moscow, 123 p. (in Russian)

Vernadsky V.I. (1924) Biosphere and Noosphere. Nauka, Moscow, 163 p. (in Russian)

Wolverton B.C., McDonald R.C., Watkins E.A.J. (1984) Foliage plants for removing indoor air pollutants from energy efficient homes. Economic Botany 38: 224-229. 